\title{
Learning to Have Fun
}

\author{
Jonathan Schaeffer \\ University of Alberta \\ Department of Computing Science \\ jonathan@cs. ualberta.ca
}

\begin{abstract}
Games have played a major role in the history of artificial intelligence research. The goal of this research largely has been to build programs capable of defeating strong human players. Most of the literature has been devoted to two-player, perfect information games-games where the research results have little wide-spread applicability. However, over the past few years the need for improved AI techniques have become apparent in commercial computer games, a $\$ 25$ billion industry. Laird and van Lent call the new generation of commercial games "AI's killer application". The buying public wants to see realistic artificial intelligence in these products. Here the the metric is a "fun" experience, not winning. Hence, the outcomes from research using these applications will be of much wider applicability. This talk will discuss the challenges of using machine learning in commercial computer games to create "fun".
\end{abstract}

